Aus der II. Medizinischen Klinik det Universitat in Budapest. (Vorstand: Hofrat Prof. Jendrassik.)

\section{Zur Aetiologie und Therapie der Ischias.}

\section{Von Dr. Josef Csiky, Assistent, z. Z. Regimentsarzt.}

Die Aetiologie der Ischias ist noch immer nicht klargestellt. In den meisten Lehrbüchern trifft man eine ganzc Reihe von ätiologischen Momenten aufgezählt, wie das schon in der älteren Pathologie der Branch war. Die Erforschung der wahren Ursachen brachte es mit sith, daß in jedem Wissenszweige - wo diese Erkenntnis möglich wurde - die durch ungenügende Kritik der Empirie aufgestellten falschen und vielseitigen Angaben auf einmal verschwanden. Wie viele Ursachen der Tabes werden in unseren älteren Lehrbüchern aufgezählt! Aber auch in den vor einigen Jahrzehnten geschriebenen Abhandlungen tiber die Aetiologie der Tuberkulose, des Abdominaltyphus, der Malaria finden wir eine große Anzahl mit bestimmter Wahrseheinlichkeit, ja sogar mit dem Gefühl der Gewißheit aufgezählte, für gleichwertig erachtete ätiologische Faktoren. Mit dieser Richtung steht unser heutiges Denken im vollen Gegensatz, und auf Grund unserer heutigen Auffassung der Krankheitsursachen ist es nötig, sämtliche noch nicht aufgcklärten Krankheiten auf ihre Actiologie und die Umstände ihrer Entstehung zu überprüfen.

Jene Grundlage unseres heutigen ätiologischen Denkens, $\mathrm{da} B$ jede echte Krankheit auf eine einzige und von den anderen verschiedene Ursache oder Gruppen von Ursachen zurückgeführt werden kann, bringt es mit sich, $\mathrm{daB}$ wir einzelne Symptomenkomplexe von echten, wirklichen Krankheiten unterscheiden können. Dic exakte Definition der Krankheit ist auch heute noch kaum möglich; als echte Krankheit können wir nur jene bezeichnen, deren Aetiologie festgestellt ist. Jedenfalls hat der $\mathrm{Satz}$, daB verschiedene Krankheitsursachen verschiedene Krankheiten verursachen, auch umgekehrt Geltung, und auf Grund anseres heutigen Denkens steht es über allem Zweifel, da B gut charakterisierte, von anderen Krankheitsbildern gut differenzierbare Krankheiten mit typischem Verlauf als echte Krankheiten betrachtet werden können und so ihre Ursache auf einheitliche, spezifische Momente zurïckgeführt werden kann. Wenn wir daher die Ursache einer Krankheit suchen, so müssen wir in erster Linie konstatieren, ob die betreffende Krankheit in jeder Beziehung als echte Krankheit aufgefaßt werden kann.

Bei den infektiösen Erkrankungen ist die Entscheidung in dieser Bezjehung nicht schwierig, auch dann nicht, wenn wir den Erreger nicht kennen. Die Variola, Scarlatina, Morbilli sind obne Zwcjel echte Krankheiten. Ein großer Teil der chemischen, also durch ein Gift veruraachten Erkrankungen bildet ebenfalls sehr typische Krankheitsformen, obwohl der oberflächliche Beobachter sehr oft nur allgemeinc Symptome findet. So verursachen z. B. die ätzenden Säuren und Laugen auf den ersten Blick nur allgemeine, nicht ganz charakteristische Symptome, aber auch in diesen Fällen bekommt die Krankheit dadurch, daß wir die krankheitserregende Ursache nachweisen, eine ganz spezifisch-charakteristische Gestalt. Und so kommt es, daß trotz Aehnlich keit der Veränderungen, die durch die Einwirkung verschiedener Giftarten entstehen, wir durch die Möglichkeit des Nachweises des be treffenden Giftes dennoch eine genaue Diagnose stellen können. In Grunde ist es dasselbe wie bei der Differentialdiagnose $z$ wischen Typhus abdominalis und Paratyphus oder anderen mit Fieber einhergehenden Erkrankungen, in denen wir die Krankheitserreger feststellen.

Viel sohwerer steht die Sache bei den auf mechanisch.traumatischer Basis entstandenen Krankhcitsbildern, obwohl wir auch hier genug charakteristisehe Formen kennen. Als häufigstes Beispiel sind hier die Herzklappenfehler zu nennen; die Trikuspidal-oder Aorteninsuffizienz oder die Stejose zählt zwar zu den cchten Erkrankungen, aber nur dann, wenn wir auch den ätiologischen Faktor in Betracht ziehen. Dieser letztere Umstand wird von den Autoren noch nicht genug hervorgehoben, es ist aber klar, daß das Wesen und die Prognose eines geheilten, au endokarditischer Basis entstandenen Klappenfehlers - sofern die Kompensation möglich ist - ganz anders ist als das weitere Schicksal eine auf arteriosklerotischer oder luetischer Basis entstandenen, fortschreitenden Klappenfehlers.

Geradeso steht es auch mit den anderen traumatischen Erkrankungen; neben den mechanischen Veränderungen muB zur richtigen Beurteilung der Erkrankung auch der ätiologische Faktor in Betracht gezogen werden. Ganz anders werden wir einen einfachen Knochenbruch beurtcilen als einen, wclcher die Folge einer Schußverletzung oder eines Tumors ist.

Dasselbe gilt von den auf mechanischem Wege entatandenen Nerven krankheiten. Dic durch den Druck eines Tumors entstandenen Symptome bedeuten nicht die Krankheit selbst, und die Natur der Erkrankung wird erst dann klar, wenn wir die Krankheitsursache oder die Natur des die Ursache bildenden Tumors festgestellt haben.

Auch bei den peripherischen Nerven finden wir dieselben Verhält. nisse: die rheumatisehe Fazialislähmung unterscheiden wir ganz be- stimmt ron den Lähmungen anderen Ursprungs; der Ausdruck Fazialis. lähmung bedeutet nur einen Symptomenkomplex nnd wird erst dunn dem Begriff ciner echten Erkrankung entsprechen, wenn wir sie als cine rhcumatische oder nach Verletzung entstandene oder durch Tumor etc. verursachte Lähmung bezeichnen.

Diese Denkweisc ging so allgemein in unsere Praxis über, dab wir in den meisten Fällen schon aus den Umständen und Symptomer auf die Ursache folgern; das bedeutet ohne $Z$ weifel soviel, daB die Ur sache eincs durch eine charakteristische, echte Frkrankung verursachten Symptomenkomplexcy einheitlich ist. So erkennen wir bei der Infiltration der Lungenspitze die Tubcrknlose, im plötzlich auftretenden, schnell wieder verschwindenden, am dritten Tagc immer wiederkehrenden Ficber dio Malaria, und wir zweifcln nicht daran, daß im ersten Falle der Kochsche Bazillus, im letzteren Falle das Malariaplasmodium die Ursache der Erkrankung bildet. Ebenso folgern wir aus dem Korsakowschen Symttomenkomplex, aus gewissen Formen der Leberzirrhose auf Alkoholintoxikation, aus gewissen Lähmungen auf Blcioder Arsenvergiftung. Aber auch die meisten Symptomenkomplexe mechanischen Ursprungs sind genügend charakteristisch; die rheumatische Fazialislälunung, die als Folge von Plattfuß entstandenen Schmerzen im FuB und anderc Krankheitsformen sind so bekannt, daB wir aus den Symptomen auch den Ursprung bestimmen körnen.

AuBer diesen Erkrankungen mit mehr oder weniger bekannter Aetiologie gibt es aber noch andere ganz bestimmte, echte Erkrankungen, die ohne Zweifel nicht infektiösen Ursprungs sind und über deren A etiologie die nueisten Autoren sich danit begnügen, alte Wahrscheinlichkeitsgründe aufzızählen. Zu dieser Gruppe gehören von den Nervenkrankheiten die multiple Sklerose, die Paralysis agitans, die Syringomyelie und noch viele andere Erkrankungen, deren Symptome, Ablauf und Natur ganz klar darauf hinweisen, daß alle diese Erkrankungen ihre spezifische, bis jetzt unbekannte Ursache haben. Die Ursache bedeutet nichts anderes als diejenigen Verhältnisse, unter denen eine Erkrankung zustandckoinmt, und wenn wir der gegenwärtigen philosophischen Tcrminologie trcu bleiben wollen, so müdsen wir diesen Ausdruck gebrauchen.

Untersuchen wir nun auf Grund dieses Gedankenganges, ob wir die Ischias als echte Erkrankung auffassen können. Nach unserer Meinung können wir auf diesc Frage mit Ja antworten, sind doch die Symptome dieses Leidens ganz besonders charakteristisch, und obwohl die Lehrbücher sehr verschiedene Gründe als ihre ätiologischen Momente anführen, wird doch jeder es für eine falsche Ischiasdiagnose halten, wenn sich als Ursache der ischiasähnlichen Schmerzen ein im Becken sitzender Tumor, eine Entzündung oder Diabetes herausstellt. In diescn Fällen hat der Kranke zwar Schnerzen, aber keine Ischias. Es hat nicht viel Sinn, wenn einzelne Autoren einen luetischen, gonor rhoischen, tnberkulösen Ursprung für inöglich oder für annehmbar halten. Das sind eben jene kritiklosen Behauptungen, die aus einem Buch in andere wandern, ohne daB für ihr Vorhandensein irgendein ernster, stichhaltiger Grund vorhanden wäre. Dahin gehört auch der Ausdruck Statique variqueuse; der Umstand, da 3 der betreffende Kranke außer seiner Frkrankung noch eine Tuberkulose, Lues, Retroflexio utcri oder Venencrweiterungen hatte, ist kei weitem kcin Argument fiir die Annahme cines ursächlichen Zusammenhanges zwischen beiden Erkrankungen.

Die Erkrankung selbst gibt in diesem Falle nicht genug Stütze zur Ergründung der Aetiologie. Ueber die durch die Ischiss verursachten anatomischen Veränderungen wissen wir kaum etwas. Die meisten Autoren wissen überhaupt garnicht, wohin sie diese Krankheit einrcihen sollen: unter die Neuralgien oder unter die Neuritisfornen. Oppenheim bringt sie sogar mit dem Muskelrheumatismus in $\mathrm{Be}-$ zichung (,,im Gefolge eines Muskelrheumatismus“ . . ., „echt myositische Prozesse können einen auf den Ischiadicus übergehenden Ent zündungsprozeß anfachen"). Anderc meinen wieder, da B sie einen

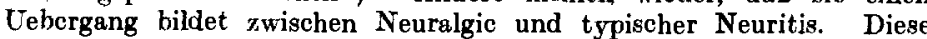
Gedanken basieren noch immer auf der alten Organozellularpathologie; sie beherrschen die einzelnen Kapitel mit noch unbekannter Aetiologie, ebenso wie in früheren Zeiten sie in der Lehre von der Tuberkulose, vom Typhus eine Rolle spielten, als die Autoren die verschicdensten ,Uebergänge“ für nıöglich hielten und beschrieben. „Der Katarrh ging in eine Entzündung, die Entzündung in Tuberkulose über." Unser heutiges pathologisches Denken geht aus dem Gedanken einer spezifischen Ursache aus, nur dieselben Umstände erzeugen dieselben Veränderungen und vice versa. Je bekannter der ätiologische Faktor einer Krankheit ist, um so weniger müssen die „Ursachen" werden.

Von diesem Grundprinzip ausgehend, müssen wir fragen, ob im Krankheitsbilde der Ischias solche Unstände nachweisbar sind, die uns zur Einsicht in dic Ursache der Erkrankung bringen können. Solche Unstände gibt cs sogar zwei: der cine ist, $\mathrm{da} B$ wir dic Analogie dieses Leidens auf einc in anderen Nervengebiet nicht finden, der andere, $\mathrm{da} ß$ es einseitig auftritt. Diese zwei Umstände müssen noch mit ein paar Worten erläutert
werden. Einzelne Autoren besprechen als Ischias auterior einen auf 
der vorderen Fläche des Oberschenkels a uftretenden Sehmerz; die klinische Beobaclitung lelirt aber, da $B$ in diesen Fallen elne ganz. andere Frkrankung zugrundrliegt, weder nach den Symptonen, noch nach dem Verlauf deckt sich das Krankheitsbild nit dem dor Ischias, soda 8 man diese Fälle garuiclıt als cchte Erkrankurngen a uffassen kanı; nach mehrfacher, griundlicher Untersuchung stellt sich bald eine ganz andere Erkrankming heraus. Einzehne Autoren befasscen sich mit der Ischias unter dem Kapitel der Neuralgien; davon aber kann iiberlakupt keine Rude sein, daß wir diesc Erkrankung mit irgendeiner Form der Neuralgie identifizieren, und die nelleren Arbeiten neigen zu derAnsich hin, sie eher als cinc Neuritis als Neuralgic aufzufassen. obwolıl es noch ıjemanden einficl, die Erkrankıng unter die iibrigen Neuritisformen einzureihen. Das ist der Grund, warum dic Ischias in jedem Lehrbuche ganz separat, in nicht entsprechender Umgebung beschricbel wird.

Die Einseitigkeit der Ischias wird von jedom Autor anerkannt; zweiseitig auftretendc Schmorzen sind nach unseren heutigen Kenntnissen kcine echte Ischias, nnd wach griindlicher, mchrmaliger Untersuchung findet man anch meistens die wahre Ursache diescr Schmerzen in einem ganz anderen pathologischen ProzeB.

Bei den akıten Erkrankungen ist dic Halbscitigkeif der Symptome ein Spiel des Zufalls, bci chronischen Erkrankungen muB inan diesem Unstande größcre Wichtigkeit beilegen.

Unter den gegebenen Verhältnissen ist der erste Unstand nur so zı erklärcn, daß mit dem $\mathbf{N}$. ischiadıcıs etwas geschehen muß, was mit einem anderen Nerv nicht geschehen kann, der zwcite Umstand zeugt wieder dafür, daß der schädliche Einflıß nur eine Seite beriihrt, oder, was viel wahrscheinlicher ist, daß er dann viel schädlicher wirkt, wenn er nur halbseitig ist. Diese Verlältnisse sind kauın anders zı erklären, als daß dic eınzige Ursache der Ischias das schlechte Sitzen ist. Diese Annahme erklärt es einzig und allcin, daß die Erkrankung nur am N. ischiadicus entsteht (oder eventilell am $\mathrm{N}$. cutaneus femoris posterior); cs ist anch selbstverständlich, daß das Körpergewicht viel stärkcre Wirkung ausübt, wenı es nur auf den einen $N$. ischiadicis einen Druck ausübt, als wenn es auf beide Seitcn verteilt wird. Wenn wir außerdem den Verlauf des Nurven und dessen Verhaltnis zum Sitzen in Betracht ziehen, so wird es sofort vorständlich, dals bci symnetıisehen Sitzen das Os ischii keinen Druck anf den Nerv ausïbeu kanu, weil dieser außerhalb des Tuber ischii verläuft und ein Druck anf dirscru nul

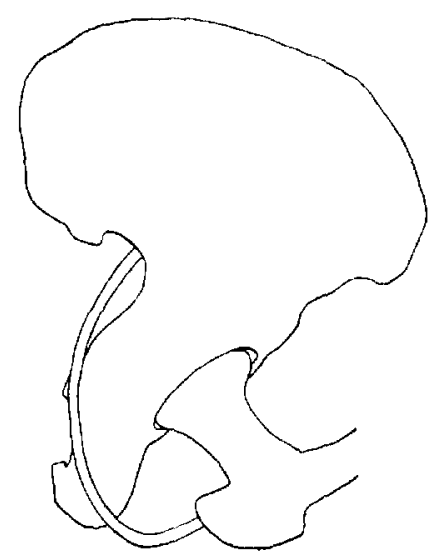

Das Becken beim Sitzen; Verbaltnis
des $\mathrm{N}$ ischiadicus zum Silzknorren. dann wirken kann, wenn das Becken noch einer Seite gebengt ist; und, wie dic beigegebene Abbllung zeigt ist hierbei eine Druckl ichtung nötig. die besonders durch die Sitzkante crmöglicht wird. Es ist wahrschein lich, da $B$ anch individuclle Momente einc Rollc spiclen, und es gibt einz.elne Menschen, bei denen der Nerv leichter zwischen dens harten Sitz. und dem Tuber ischii gedrückt wird.

Das symmetrische Sitzen wird a ber nicht bci jedcr Beschäftıging eingchalten. So bengen sieh dic: incisten Mensclien bcion Schrciben nacli vorn und anf die Seite, und weml dazıl noch der Rand des Stulıles, auf den der Botreffende sitzt, liart nud kantig ist, so ist es schr leiclit begrciflich, dis anf don N. ischiadiens cin Druck ansgeübt wırd. Es gibt aber noclı cinen anderen Umstand, bci welchen diescr Druck noch intensiver ist, und das ist das Sitzen wihrend des Stullabganges auf harter, enger Basis nach vorn nud meistens anch stark(r anf eine Seite gebengt. Fs ist auch nicht so selten, da B das bekannte Kribbelgefiill im betrcffenden Bein (das sogenannte Einschlafen des Beines) durch diesen Druck verursacht wird. Dieser Zustand ist vorübergchend, wie eine akute Ischias, es ist aber wahrscheinlich, da $B$ die echte Ischias durch den öfters wicderkchrenden, aber nieht so intensiven Druck verursacht wird. Die allf die Nerven einen Druck ausübenden Tumoren verursachen bekanntlich einen sich auf den ganzen Nerv ausbreitenden Schmerz, und diese Analogie wirft etwas Licht anf dic Erkrankıı. Das häıfig wiederkehrcnde Traumı verursacht höchstwahrsehcinlich eineı neıriti schen Prozeß in Nerven.

Diese Erkenntnis hat auch praktische Folgen. In erster Rc.ihe dic, da $\beta$ man solche Kranke auf diese Unıstände anfmerksanı machen nuß; sie miissen ihren gewöhılichen Schrcibstuhl anstanschen nud dïrfen nur auf eincm Stuhl sitzen, auf wclchem sic einem solchen Druck nicht ausgesetzt sind. Patient soll auf das symmetrisclic Sitzen acht geben, wcil der Nerv gerade hierdurch am besten rom Druck bewahrt blcibt. Man muß ferner den Kranken alıch auf den richtigen Sitz am Klosett aufmerksam machen. Dies alles ist deshalb so wichtig, weil nicht jener Druck gefährlich ist, der cinen sofortigen Schmerz verursacht, sondern besonders derjenige, der innerhalb dieser Gronzt: bleikt; gegen den star- keren Druck verwahrt sich ohnedies jeder. Aneh an anderen Körperstellen entstelien infolge regelı̈̈ßig wiederloolten Drucks schmerzhafte Anschwollungen.

A uf dicser Grundlage miissen wir natirlich in Abrede stellen, daß dic Ischias an verschicdenen Stellen des Nerven entstehen kann; inserer Meinung nach ist der Ausgangspunkt innuer jene Stelle, welche in der Nachbarsehaft des Tuber ischiadiens ist. Disse Stclle findet man am besten, wenn sieh der Kranke beim Liegen auf die gesunde Seitc dreht, das kranke Bein etwas anzieht und man dabci nun ncben den Trochanter major mit senkrecht gehaltenen Fingern eingeht und hierbei einen Druek gegen den Tuber ischiadicus ansïbt. Dies ist die bestc Metlıode zur Bestimnuung des Druckpunktes am Ischiadicus. Es gibt auch kcinen crnsten Grund dafür, da $B$ in gewissen Fülen der A usgangspounkt der Erkrankung in dic Riickenmarkswurzeln zu lokalisieren wäre; ebcnsowenig kann als Ursache dic Obstipation gelten (wic das frïher besonders Gussenbauer behauptete); diescr lctzterc Unstand kënntc höchstens insoweit cinen Einflı $B$ liaben, da $B$ bei Obstipation ein lingeres Sitzen notig ist auf dem ohnehin schädlichen Klosettsitz. Unsere Kranken werden seit Jahren nacl dieser Richting ausgefragt, und ungere Erfahrung ist, daß dic Obstipation bei diesem Lciden nicht so häufig vorkommt und da $B$ Abfiihrmittel keinen nennenswerten Einflıß anf die Erkrankung laben. Hingegen gelang es mehrmals, eine im Anfangsstadium befindliche Ischias dadırch enm Stillstand zu bringen, da $B$ wir dem Kranken ein entsprechendes Sitzen empfalılen. Auf Grund unserer Erfahrung ist es zweifcllos, daß viele Kranke deslialb ihr Leiden nicht los werdell, weil sic alf diesen wichtigen Umstand nicht alfmerksain gemacht werden.

Zuletzt will ich noeh bemerken, daß wir anf der Klinik seit Jahren die Erkrankıngen mit dem Plätteisen behandeln. Die Mehrzahl unserer Kranken versuchte vergebens die verschiedenen Thermalkuren und Bäder. Die Heilung solcher Kranken gelang in den nieisten Fällen ohnc heiße Bäder, nur durch Plätten. Die Technik dieses Verfahrenk, das, wic es schcint, in der häuslichen Behandlung schon länger bckannt ist, ist folgende. Der Kranke liegt auf dem Banche oder - wie wir es als cine fïr die Untersuchung des schmcrzhaften Nerven günstigste Lage beschrieben - - auf der gesunden Scite, wobei er scin Knie anzieht. In solcher Lage konmnen wir dein erkrankten Nerven noch nàher als in Banclsage. Dcr Obcrsclenkel wird nun mit einem cinfachen oder doppelten Lcintuch zugedeckt. Wir nchmon danu zwei stärkere Decken und legen sic derart allf den Kranken, daß die eine von oben bis zum Obcrsclicnkel, dic zweite hingegen von lier nach abwärts das Bcin warm halt. Dic zwei Decken konmen an der Austrittsstelle des N. ischiadicus zusammen. Da beginnen wir das Platten, indem wir das warme Eisen zwischen den beiden Decken auf das Leintuch iibor der kranken Stelle anbringen. Wichtig ist bei diesen Verfahren, da $B$ das Eisen die richtige Tempcratır liat; zı diesem Zwccke ist an geeignetsten ein elektrisches Platteiscr, weil wir dessen Tenperatur durch einen dazwischen geschalteten Widerstand beliebig cinstellen können. Anch nuit gewöhnlichem Eisen ge:lingt die Kur natürliclı. Diejenige Temperatır ist am zweckmäßigsten, bei welclicr es möglich ist, das Eisen cine halbe bis ganze Minnte an einer Stelle zu halten: das Wärmegefühl muß aber sehr intensiv und zulletzt schon unerträgliclı sein. Dann rückt man mit einer Plittcisenbrcitc weiter, riclutct abcr die Decken inıner so, daß der Krankc an den iibrigen Stellen überall zugedeckt sein soll und der soeben bestralıltc Teil sofort unter die Derke komnt, damit es noch längere Zeit unter der Wirknng dor Warme bleibe. Die Wärme des Eisens ist eine selır intensiv strahlende, und wenn wir die Temperatur gut wählen, so dringt die Wärmew irkung sehr tief ein. Gewiß steigert das Resultat les Verfahrens, daß der Kranke anf kleiner Fläche alf dicse Art viel nnchr Wärme aısshält, als wenn die Wärne inı Bad auf den ganzen Körper cinwirkt.

Allf diesc Wcise behandeln wir die ganze schmerzhafte Gegend cntilung des Beines, was ungcfähr zehn Minuten crfordert, obwohl es vielleicht überflïssig ist, den ganzen Nerv zll behandeln, da der Ort der Veränderung an der Gegend der Glutäalfalte ist. Diese Behandlung verträgt der Kranke nieistens rocht gut, man wiederholt sie täglich, ansgenommen, wenn sich die Empfindlichkeit des Kranken gegen die Wärme stcigert. In solchen Falle macht man ein bis zwei Tage Pause. Ill algemeinen nuß man im Anfang vorsichtiger sein, da der Nerv empfindlicher ist und auf mildere Behandlung die Besserung eher eintritt, als wenn wir zu starke Hitze anwenden.

Zusammenfassung. Die Ischins wird dadurch hervorgerufen, da B $\mathrm{m}^{*} \cdot \mathrm{l}$ bein Sitzen - besondcrs auf ungeeigneter Sitzfläche - den Ner. ven an den Knoc'1'n andrïckt. Do.r Druckpunkt läßt sich am genauesten in der Seitenlage fotstellen, wenn nan um den Trochanter den leicht palpablen Verlanf des Nerven folgt. Zurr Bchandlung eignet sich sęhr gut das Verfahren mit dern Plätteisen. 